\title{
SOFTWARE FOR MODELLING AND SIMULATION OF A REMOTELY-OPERATED VEHICLE (ROV)
}

\author{
Chin, C. S.; Lau, M. W. S.; Low, E. \& Seet, G. G. L. \\ Robotic Research Centre, Department of Mechanical and Aerospace Engineering, \\ Nanyang Technological University, Singapore 639798 \\ E-Mail: chin0014@ntu.edu.sg
}

\begin{abstract}
This paper considers the dynamic modelling and simulation of an underactuated Remotely Operated Vehicle (ROV) designed by Robotic Research Centre (RRC), in Nanyang Technological University (NTU) using a ROV Design and Analysis (RDA) toolbox written in MATLAB/SIMULINK ${ }^{\mathrm{TM}}$. The proposed RDA provides a means to simulate the mathematical models of ROV and the control system design before planning for final hardware implementation. This is especially useful, as the ROV model is highly uncertain due to the hydrodynamic forces that are nonlinear and coupled. However, there is no commercial software for ROV control system design and analysis.

(Received in May 2006, accepted in August 2006. This paper was with the authors 1 month for 1 revision.)
\end{abstract}

Key Words: Remotely Operated Vehicle, Modelling, Simulation, MATLAB/SIMULINK ${ }^{\mathrm{TM}}$

\section{INTRODUCTION}

The Remotely Operated Vehicle (ROV) designed by Robotic Research Centre (RRC), RRC ROV II is designed to be a sub-compact intervention class ROV combining the functions of an eye-ball ROV and a work class ROV. It has a man-in-the-loop as the supervisor with complementary on-board controller for local control and decision making capabilities. The vehicle is equipped with a suite of navigational sensors; INS, Doppler and side scan sonars- a pair of cameras with "stereo viewing" capabilities. Its designed tasks include inspections and repairs of pipelines and structures. Station-keeping would require the vehicle to hover over a certain set of coordinates while maintaining a fixed orientation while tracking require the ROV to follow the trajectory of the pipeline in the presence of various types of disturbances.

This control of this task is exacerbated by the vehicle having non-linear dynamics, underactuated and motion amongst its DOF is coupled. By using the ROV Design and Analysis (RDA) simulation software, it helps to simulate the ROV control system in the best-toknowledge simulation environment before actual implementation for sea trial. It is flexible enough to extend to other type of ROV instead of RRC ROV II. To best of our knowledge, there are no commercial ROV modelling and simulation toolboxes for control system design application and analysis. Except the Marine Systems Simulator (MSS) toolbox [1] that was developed by T. I. Fossen and his students as a supporting tool for his courses on guidance and control of ships and his book [1]. But its application is mainly for marine vessel application and may not be suitable for submerged vehicle such as remotely operated vehicle (ROV) and analysis package is not available. The outline of this paper is as follows: A brief introduction of the modelling of ROV. Followed by, an overview of the RDA software characteristic and organization. Lastly, some concluding remarks.

\section{GENERAL ROV MODEL OF RRC ROV II}

A general dynamic model formulation for general underwater robotic vehicle $[2,5]$ is used and often found to be quite good for many underwater applications. Detailed derivation of the 
parameters used in (1) can be found in [1]. The general motion of a ROV can be described by using a body-fixed frame relative to an earth fixed frame (see Fig. 1) and can be expressed in a more compact form as:

$$
M \dot{\mathrm{v}}+C(\mathrm{v}) \mathrm{v}+D(\mathrm{v}) \mathrm{v}+g(\eta)=\tau
$$

where $M=M_{R B}+{ }$. $M_{A} \in \mathfrak{R}^{6 \times 6}$ is the sum of mass inertia and added mass matrix; $C=C_{R B}+C_{A}$ $\in \mathfrak{R}^{6 \times 6}$ is the sum of Coriolis and centripetal and added forces and moments matrix; $D \in \mathfrak{R}^{6 \times 6}$ is the diagonal hydrodynamic forces and moments matrix; $g \in \mathfrak{R}^{6}$ is the vector of gravitational and buoyancy restoring forces and moments vector; $\tau=T \bar{u} \in \mathfrak{R}^{6}$ is the summation of all force and moment inputs vector with $T \in \mathfrak{R}^{6 \times 4}$ is the thruster configuration matrix written as:

$$
T=\left[\begin{array}{cccc}
1 & 1 & 0 & 0 \\
0 & 0 & \sin \beta & -\sin \beta \\
0 & 0 & \cos \beta & \cos \beta \\
0 & 0 & -\delta & \delta \\
-\varepsilon & -\varepsilon & -\alpha \cos \beta & -\alpha \cos \beta \\
\gamma & -\gamma & \alpha \sin \beta & -\alpha \sin \beta
\end{array}\right]
$$

where $\alpha=0.017 \mathrm{~m}, \beta=45^{\circ}, \gamma=0.31 \mathrm{~m}, \delta=0.293 \mathrm{~m}, ; \varepsilon=0.016 \mathrm{~m} ; \bar{u} \in \mathfrak{R}^{6}$ is a voltage input vector; $\quad \mathrm{v}=\left[\mathrm{v}_{1}, \mathrm{v}_{2}\right]^{T} \in \mathfrak{R}^{6}$ are the linear and angular velocities vector consisting $\mathrm{v}_{1}=[u, v, w]^{T}$ and $\mathrm{v}_{2}=[p, q, r]^{T}$ respectively; $\eta=\left[\eta_{1}, \eta_{2}\right]^{T} \in \mathfrak{R}^{3} \times S^{3}$ are the positions and orientation angles vector consisting $\eta_{1}=[x, y, z]^{T}$ and $\eta_{1}=[\phi, \theta, \psi]^{T}$ respectively. The kinematics equations or Euler transformation between the body and earth frames can be written as:

$$
\begin{aligned}
\dot{\eta} & =J\left(\eta_{2}\right) \mathrm{v} \\
& =\left[\begin{array}{cc}
J_{1}\left(\eta_{2}\right) & 0 \\
0 & J_{2}\left(\eta_{2}\right)
\end{array}\right] \mathrm{v}
\end{aligned}
$$

where $J_{1}\left(\eta_{2}\right)$ and $J_{2}\left(\eta_{2}\right)$ are:

$$
\begin{gathered}
J_{1}\left(\eta_{2}\right)=\left[\begin{array}{ccc}
c(\psi) c(\theta) & -s(\psi) c(\phi)+c(\psi) s(\theta) s(\phi) & s(\psi) s(\phi)+c(\psi) c(\phi) s(\theta) \\
s(\psi) c(\theta) & c(\psi) c(\phi)+s(\phi) s(\theta) s(\psi) & -c(\psi) s(\phi)+s(\theta) s(\psi) c(\theta) \\
-s(\theta) & c(\theta) s(\phi) & c(\theta) c(\phi)
\end{array}\right] \\
J_{2}\left(\eta_{2}\right)=\left[\begin{array}{ccc}
1 & s(\phi) t(\theta) & c(\phi) t(\theta) \\
0 & c(\phi) & -s(\phi) \\
0 & s(\phi) / c(\theta) & c(\phi) / c(\theta)
\end{array}\right]
\end{gathered}
$$

respectively and $s=\sin (),. c=\cos (),. t=\tan ($.$) . Note that this transformation is undefined for$ $\theta= \pm 90^{\circ}$. To overcome this singularity, a quaternion approach must be considered. However, in the project this problem does not exist because the vehicle is not required to operate on $\theta= \pm 90^{\circ}$. Moreover, the vehicle is completely stable in roll and pitch, and the thruster actuation is not enough to move the vehicle to operate at this angle. The parameters used in equation 1 and 3 are defined in Table I. In this paper, all ROV parameters used were extracted from RRC ROV II [3, 4]. 
Table I: Notations used in ROV.

\begin{tabular}{|c|c|c|c|}
\hline DOF & Motion Descriptions & $\begin{array}{c}\text { Positions and } \\
\text { Orientations }\end{array}$ & $\begin{array}{l}\text { Linear and } \\
\text { Angular } \\
\text { Velocities }\end{array}$ \\
\hline 1 & $\begin{array}{l}\text { Motions in the } x \text { - } \\
\text { direction(surge) }\end{array}$ & $x$ & $u$ \\
\hline 2 & $\begin{array}{l}\text { Motions in the } y \text { - } \\
\text { direction(sway) }\end{array}$ & $y$ & $v$ \\
\hline 3 & $\begin{array}{l}\text { Motions in the z- } \\
\text { direction(heave) }\end{array}$ & $Z$ & $w$ \\
\hline 4 & $\begin{array}{l}\text { Rotations about the } \\
x \text {-axis(roll) }\end{array}$ & $\phi$ & $p$ \\
\hline 5 & $\begin{array}{l}\text { Rotations about the } \\
y \text {-axis(pitch) }\end{array}$ & $\theta$ & $q$ \\
\hline 6 & $\begin{array}{l}\text { Rotations about the } \\
\text { z-axis(yaw) }\end{array}$ & $\psi$ & $r$ \\
\hline
\end{tabular}

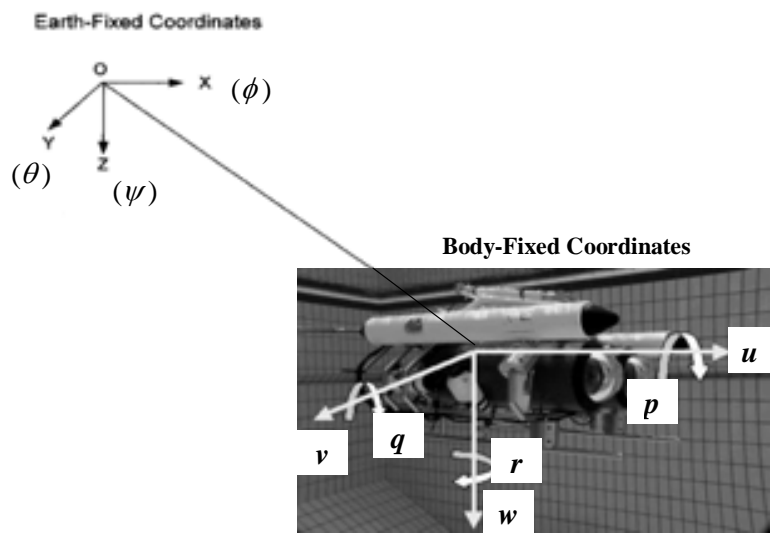

Figure 1: Experimental RRC ROV II.

For simplification and practicality consideration, the body-fixed reference frame was chosen to be the gravity centre, i.e. $x_{G}=y_{G}=z_{G}=0$. This leave the $M_{R}$ to be block diagonal where the first $3 \times 3$ block consists of ROV's mass and second block consisting of inertia term of the same size. In this second block, the off-diagonal elements of inertia term $I_{x y}, I_{x z}$ and $I_{y z}$ in $M_{R}$ are much smaller than the diagonal counterparts. Instead of getting all the thirty-six added mass parameters, the diagonal approximation is found to be quite good for many applications especially when the ROV is moving at low speed. By computing the added mass coefficient used in $M_{A}$ (and $C_{A}$ ) by principle of Strip theory [2], the added mass coefficients for each directions $\left(X_{\dot{u}}=-0.6, Y_{\dot{v}}=-107, Z_{\dot{w}}=-107, K_{\dot{p}}=-0.0023, M_{\dot{q}}=-6.23\right.$ and $N_{\dot{r}}=-6.23$ ) are computed. As a result, the total mass inertia in (1) becomes:

$$
M=\left[\begin{array}{cccccc}
m-X_{\dot{u}} & 0 & 0 & 0 & 0 & 0 \\
0 & m-Y_{\dot{v}} & 0 & 0 & 0 & 0 \\
0 & 0 & m-Z_{\dot{w}} & 0 & 0 & 0 \\
0 & 0 & 0 & I_{x x}-K_{\dot{p}} & -I_{x y} & -I_{x z} \\
0 & 0 & 0 & -I_{y x} & I_{y y}-M_{\dot{q}} & -I_{y z} \\
0 & 0 & 0 & -I_{z x} & -I_{z y} & I_{z z}-N_{\dot{r}}
\end{array}\right]
$$

where the mass, $m=113.2 \mathrm{~kg}$ and inertia $I$ terms are obtained from the computer-aided design software, PRO-E. As compared to [3], the improved moment of inertia value with an addition float become: $I_{x x}=6.100 \mathrm{~kg} \cdot \mathrm{m}^{2}, I_{y y}=5.980 \mathrm{~kg} \cdot \mathrm{m}^{2} I_{z z}=9.590 \mathrm{~kg} \cdot \mathrm{m}^{2}, I_{x y}=-0.00016 \mathrm{~kg} \cdot \mathrm{m}^{2}, I_{x z}=-$ $0.185 \mathrm{~kg} . \mathrm{m}^{2}$ and $I_{y z}=0.0006 \mathrm{~kg} \cdot \mathrm{m}^{2}$. As observed on the ROV, it has few planes of symmetry. 
The highest $I$ term (correspond to the most symmetry plane) are the $X Z$ plane, $Y Z$ plane and followed by $X Y$ plane.

$C(\mathrm{v})=C_{R}(\mathrm{v})+C_{A}(\mathrm{v}) \in \mathfrak{R}^{6 \times 6}$ is the Centripetal and coriolis matrix including added mass term $C_{A}(\mathrm{v})$ and rigid body term $C_{R}(\mathrm{v})$ :

$$
C(\mathrm{v})=\left[\begin{array}{cc}
O_{3 \times 3} & C_{12}(\mathrm{v}) \\
-C_{12}^{T}(\mathrm{v}) & C_{22}(\mathrm{v})
\end{array}\right]
$$

with

$$
\begin{gathered}
C_{12}(\mathrm{v})=\left[\begin{array}{ccc}
m\left(y_{G} q+z_{G} r\right) & -m\left(x_{G} q-w\right)-Z_{\dot{w}} w & -m\left(x_{G} r+v\right)+Y_{\dot{v}} v \\
-m\left(y_{G} p+w\right)+Z_{\dot{w}} w & m\left(z_{G} r+x_{G} p\right) & -m\left(y_{G} r-u\right)-X_{\dot{u}} u \\
-m\left(z_{G} p-v\right)-Y_{\dot{v}} v & -m\left(z_{G} q+u\right)+X_{\dot{u}} u & m\left(x_{G} p+y_{G} q\right)
\end{array}\right] \\
C_{22}(\mathrm{v})=\left[\begin{array}{ccc}
0 & -I_{y z} q-I_{x z} p+I_{z} r-N_{\dot{r}} r & I_{y z} r+I_{x y} p-I_{y} q+M_{\dot{q}} q \\
I_{y z} q+I_{x z} p-I_{z} r+N_{\dot{r}} r & 0 & -I_{x z} r-I_{x y} q+I_{x} p-K_{\dot{p}} p \\
-I_{y z} r-I_{x y} p+I_{y} q-M_{\dot{q}} q & I_{x z} r+I_{x y} q-I_{x} p+K_{\dot{p}} p & 0
\end{array}\right]
\end{gathered}
$$

where gravity centre, i.e. $x_{G}=y_{G}=z_{G}=0$.

$D(v) \in \mathfrak{R}^{6 \times 6}$ is the hydrodynamic damping matrix consisting of a linear and quadratic terms. Potential damping $[2,6]$, wave drift damping, skin friction and vortex shedding damping cause the main damping on the ROV. However, the contribution from the potential and wave drift are negligible as compared to vortex shedding damping and skin friction. The viscosity of the fluid mainly contributes these damping effects. Usually, these viscous damping on the underwater vehicles are nonlinear (or quadratic) and coupled in nature. Nevertheless, if the system has planes of symmetry, the terms higher than second-order are negligible and the vehicle is performing a non-coupled motion, the diagonal approximation [2] is found to be quite good for many applications.

$$
D=-\operatorname{diag}\left\{X_{u}+X_{u|u|}|u|, Y_{v}+Y_{v|v|}|v|, Z_{w}+Z_{w|w|}|w|, K_{p}+K_{p|p|}|p|, M_{q}+M_{q|q|}|q|, N_{r}+N_{r|r|}|r|\right\}
$$

where the quadratic damping coefficients of RRC ROV II for each DOF are:

$$
\begin{aligned}
& X_{u|u|}=-423 N \cdot m^{-2} s^{2}, Y_{v|v|}=-747 N \cdot m^{-2} s^{2}, Z_{w|w|}=-735 N \cdot m^{-2} s^{2} K_{p|p|}=-99 N \cdot m^{-2} s^{2}, \\
& M_{q|q|}=-126 N \cdot m^{-2} s^{2} \text { and } N_{r|r|}=-62 N \cdot m^{-2} s^{2} .
\end{aligned}
$$

and the estimated linear drag coefficients of the RRC ROV II are:

$$
\begin{aligned}
& X_{u}=-252.98 N \cdot m^{-2} s^{2}, Y_{v}=-1029.51 N \cdot m^{-2} s^{2}, Z_{w}=-1029.51 N \cdot m^{-2} s^{2}, K_{p}=-97.78 N . m^{-2} s^{2}, \\
& M_{q}=-142.22 N \cdot m^{-2} s^{2} \text { and } N_{r}=-71.11 N \cdot m^{-2} s^{2} .
\end{aligned}
$$

Lastly, $\mathrm{g}(\eta) \in \mathfrak{R}^{6}$ is the gravitational and buoyancy vector:

$$
g\left(\eta \left(=\left[\begin{array}{c}
(W-B) \sin \theta \\
-(W-B) \cos \theta \sin \varphi \\
-(W-B) \cos \theta \cos \varphi \\
-\left(y_{G} W-y_{B} B\right) \cos \theta \cos \varphi+\left(z_{G} W-z_{B} B\right) \cos \theta \sin \varphi \\
\left(z_{G} W-z_{B} B\right) \sin \theta+\left(x_{G} W-x_{B} B\right) \cos \theta \cos \varphi \\
-\left(x_{G} W-x_{B} B\right) \cos \theta \sin \varphi-\left(y_{G} W-y_{B} B\right) \sin \theta
\end{array}\right]\right.\right.
$$

where $W$ and $B$ is the gravitational and buoyancy force respectively. The $x_{B}, y_{B}$ and $z_{B}$ refer to the center of buoyancy. The vehicle is designed to be neutrally buoyant in the water, that is, the gravity force equal to the buoyancy force $(W=B=1110.5 N)$. The center of buoyancy $z_{B}$ will be located directly above the center of gravity $z_{G}\left(x_{B}=x_{G}, y_{B}=y_{G}, z_{B}-z_{G}=0.048 \mathrm{~m}\right)$. 


\section{RDA CHARACTERISTICS AND ORGANIZATION}

After having the ROV model, the next step is to perform the simulation using the RDA toolbox. Prior to that, the RDA characterization and organization are explained. The RDA is organized as a set of SIMULINK ${ }^{\mathrm{TM}}$ libraries, GUIs and MATLAB ${ }^{\mathrm{TM}}$ support functions:

- Analysis,

- Identification

- Propeller

- ROV Controller Design Applications

- Thruster Controller Design Applications

- ROV Model

- Scope Utilities

The RDA toolbox incorporates the SIMULINK ${ }^{\mathrm{TM}}$ library, and its associated modules such as the MATLAB ${ }^{\mathrm{TM}}$ functions. In the following, we will describe each of the modules. Each module consists of SIMULINK ${ }^{\mathrm{TM}}$ blocks are elementary constitutive parts of the RDA SIMULINK ${ }^{\mathrm{TM}}$ libraries and are used to create other complex blocks or models or both. A masked block serves to establish a boundary for modular modelling approach. The new block is named and masked. Under the mask, the following color codes are used for easy recognition of elementary block function:

- Green- input port

- Red-output port

- Yellow-others

- Orange-model info

- Grey- MATLAB ${ }^{\mathrm{TM}}$ function, GUI and others

- Cyan-masked block

\subsection{Analysis Module}

This Analysis library allows the user to perform linear analysis of an open loop system such as the RRC ROV II. However, it can be applied to other ROV model. The analysis involves the checking of the open-loop stability, transmission zeros, state controllability and observability and condition number of the system. The bandwidth of the ROV (a multi-inputsmulti-outputs type) is determined using singular values. Gershogrin discs can be plotted to shown the level of states coupling. Detail explanation of each analysis can be found in [15].

\subsection{Identification Module}

As shown in Fig. 2, the system under test is a thruster. However it can be the ROV or any other model. By double clicking on the colored boxes, the function stated in the box is activated. This frequency analyzer is capable to overcome the problem of nonlinear distortion and noise corruption in the measurement scheme as shown in Fig. 3 in which the measured output $y(t)$ is first multiplied by sine and cosine respectively and then integrated. Notice the twin processes of multiplication and averaging which are present in all noise suppressing identification and estimating techniques. Effectively the setup of Fig. 3 correlate the output $y(t)$ with different versions of the input.

The frequency analyzer function in Fig. 3 is briefly explained as follows. The measured system output is multiplied by both sine and cosine of the test frequency, $f$. The results of the multiplication are then fed into two identical integrators where they are averaged over $T$ seconds. As the average time increases, the contribution of all unwanted frequency 
component in $y(t)$ got zero and the integrator output $R(t)$ and $I(t)$ become constant values that depends on the gain and phase of the system transfer function at the test frequency. In practice, the averaging is conducted over a finite time interval $T$ and it is necessary for the reason that $T=N \times p i / f$ be made an integer multiple of the test frequency period. The input is applied to the system for a number of cycles, $N$ to allow the transients to die away before the integration starts.

\subsection{Propeller Model Module}

This propeller model library consists of different type of thruster model derived under different assumption. For the flow notations, the flow through the propeller is named as propeller axial flow, $u_{p}$ while flow outside this boundary is known as simply axial flow, $u_{a}$. Detail and its derivation can be found in $[7,8]$. The analysis in this module involves the following cases:

- Taking into the account of propeller axial flow dynamic, $u_{p}$ and its variation in axial flow dynamic, $u_{a}$. Electrical and rotating propeller shaft dynamic are considered.

- Taking into the account of axial flow dynamic, $u_{a}$ only. Electrical and rotating propeller shaft dynamic are considered [7, 8].

- Without considering the axial flow dynamic, $u_{a}$ and $u_{p}$. Electrical and rotating propeller shaft dynamic are considered only.

- Consider the axial flow dynamic, $u_{a}$ using advance number, $J_{a}$. Electrical and rotating propeller shaft dynamic are not considered.

- Approximate the flow dynamic, motor electrical and shaft dynamic as a first-order transfer function.

- Approximate the electrical and rotating propeller shaft dynamic as first order transfer function and axial flow is not considered.

For completeness, the propeller, flow, shaft and motor parameters used in the simulation are listed in the model information box. To facilitate smooth simulation, the user is advised to use the ODE23 solver of variable step size and the relative tolerance of 1e-3.

\subsection{ROV Controller Design Application Module}

This ROV controller design library contains various ROV controller design. In controller design, it is commonly accepted that linear PID (or PD) controller based on linearized model does not perform as well as sliding mode control (SMC) [12], adaptive control (AC) [10] and feedback linearization (FBLN) [13], amongst others, for instance in trajectory tracking and when uncertainties are present [9]. It has been shown that $\mathrm{AC}$ can be designed to be robust. Uncertainty can also be designed using robust controller such as the $\mathrm{H}_{\infty}$ methodology [11]. However, PID/PD controllers are commonly used in commercial ROV, as it is easily understood amongst pilots. To overcome certain limitations, they can be used in combination, for example, with AC dynamic compensation [14]. The controller designs selected in this module are:

- Cascade control using nonlinear PD controller for heading (outer loop) and thruster allocation for inner loop.

- Hybrid control combining the passivity and sliding mode control.

- Nonlinear PID sliding mode control.

- Nonlinear Lyapunov-based PID controller design.

- PID controller. 


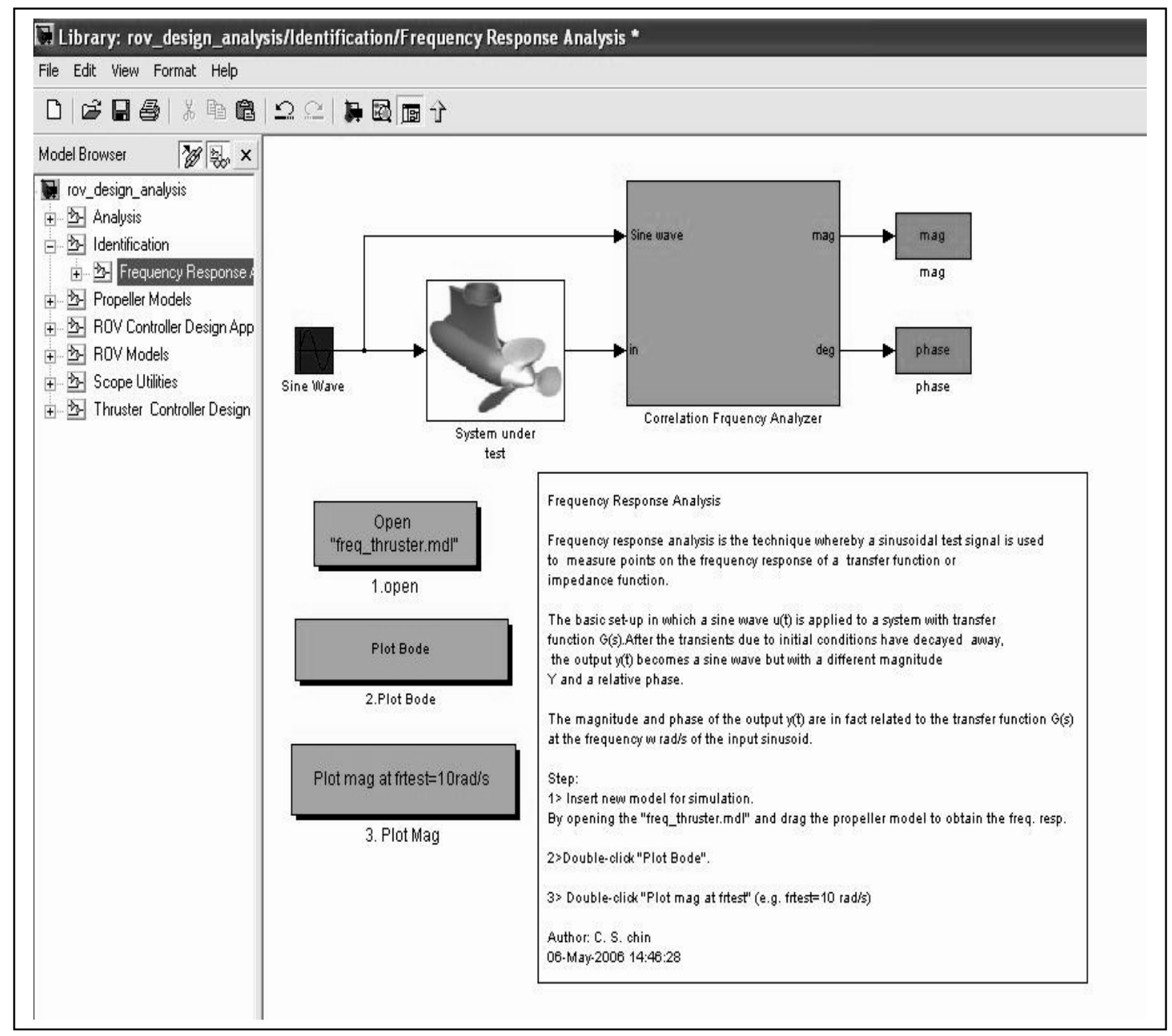

Figure 2: SIMULINK ${ }^{\mathrm{TM}}$ Library Browser Showing Identification Module.

All controller designs use the RRC ROV II model (except for the last item that uses a semi-submerged vessel). Often controller parameters are tuned until a satisfactory performance is achieved in term of the steady and transient response of the ROV's positions.

As shown in Fig. 5, the block diagram of the PID controller was built using the available blocks in each module. Performance of the PID control scheme has been investigated in the RDA toolbox. Notice that the Euler transformation block and other utilities block can be conveniently copy and paste on this block diagram for simulation. Prior to simulation, simulation parameters need to be set. The simulation time of 100s, ODE45 solver with variable step size and a relative tolerance of 1e-3 were set. The RRC ROV II parameters used in simulation can be found in Section 2. As the vehicle is currently fitted only with limited sensors, the desired position commands values, $\eta_{d}=\left[\begin{array}{llllll}0.5 & 0 & 1 & 0 & 0 & 0\end{array}\right]^{T}$ are chosen with this purpose in mind. The objective is to regulate the position of the RRC ROV II to $x=0.5 \mathrm{~m}$ and $\mathrm{z}=1 \mathrm{~m}$ or the error signal equal to zero. The PID was selected due to its simplicity in implementation and it's widely used in control application. The control algorithm requires little computing resources, and is therefore suitable for an on-board implementation.

As observed, the states are separated into actuated and unactuated states. The former uses the PID controller while the later is left uncontrolled, since the unactuated states are selfstabilized. Varying the mass inertia and Coriolis and centripetal matrix within their lower and 
upper limit tested the robustness of the PID controller. This can be modelled as a twodimensional (2D) lookup table as shown in Fig. 5. During simulation, only the maximum value (or worst-case perturbation) is used. Similarly the perturbation for the centripetal and coriolis and hydrodynamic damping matrix was set as: $C \leq 0.001\|L\|+0.001$ where $L=\left[\begin{array}{ll}\eta-\eta_{d} & \dot{\eta}-\dot{\eta}_{d}\end{array}\right]^{T}$. PID control parameters were obtained from the Response Optimization (using gradient descent method) toolbox in SIMULINK and results in: $K_{p}=$ diag $\{11,11,9,1\}, K_{i}=\operatorname{diag}\{2,4,1,2\}$, and $K_{d}=\operatorname{diag}\{1,1,2,1\}$. As observed in Fig. 6, the output approaches the reference input, $x=0.5 \mathrm{~m}$ and $z=1 \mathrm{~m}$ under the parametric perturbation. The surge position response is quite oscillatory as shown in Fig. 6.

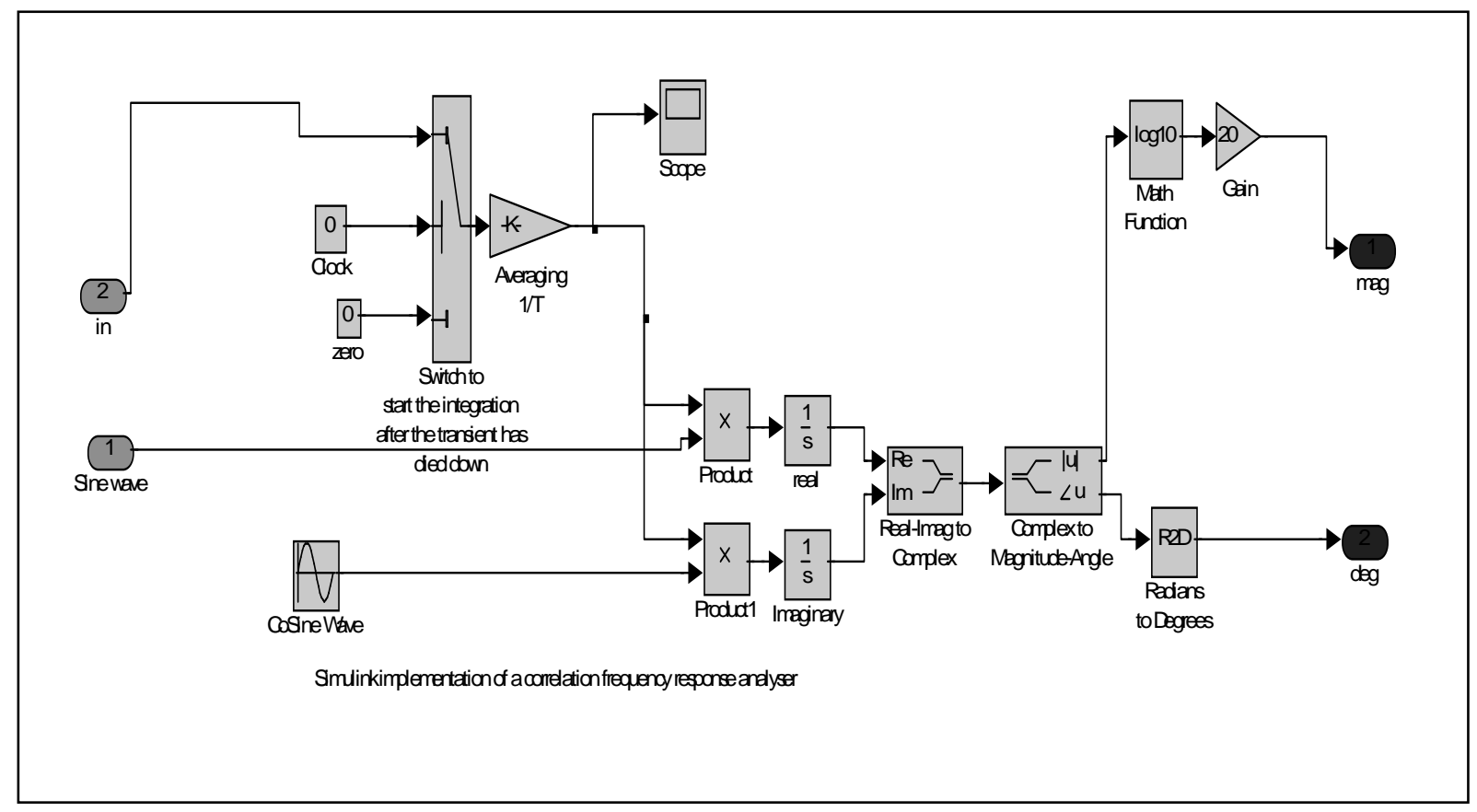

Figure 3: Example of the Frequency Analyzer.

\subsection{ROV Model Module}

As shown in Fig. 4, the ROV model library contains various ROV models. The RRC ROV II model parameters are obtained in the Section 2. The function blocks as shown in Fig. 7 can represent each row in (11). This allows series of matrix to be solved numerically. In Fig.8, a graphical user interface (GUI) was written to determine the ROV RRC II parameters. Documentations are included to explain the derivation and parameters used in the calculation. The tether forces with and without external disturbances are plotted. Note that only the one without external disturbance is plotted on the panel as shown in Fig. 8. The rest is shown in separate figures for easy manipulation. By performing the "Basic Fitting" under the menu bar "Tool", equation representing the graph can be computed. The ROV models available for simulations are the followings:

- $\quad$ RRC ROV 2

- Naval Postgraduate School (NPS) AUV

- Deep Submerged Rescue Vehicle (DSRV)

Besides some utilities block involving thruster configuration are provided as part of the ROV modelling module. 


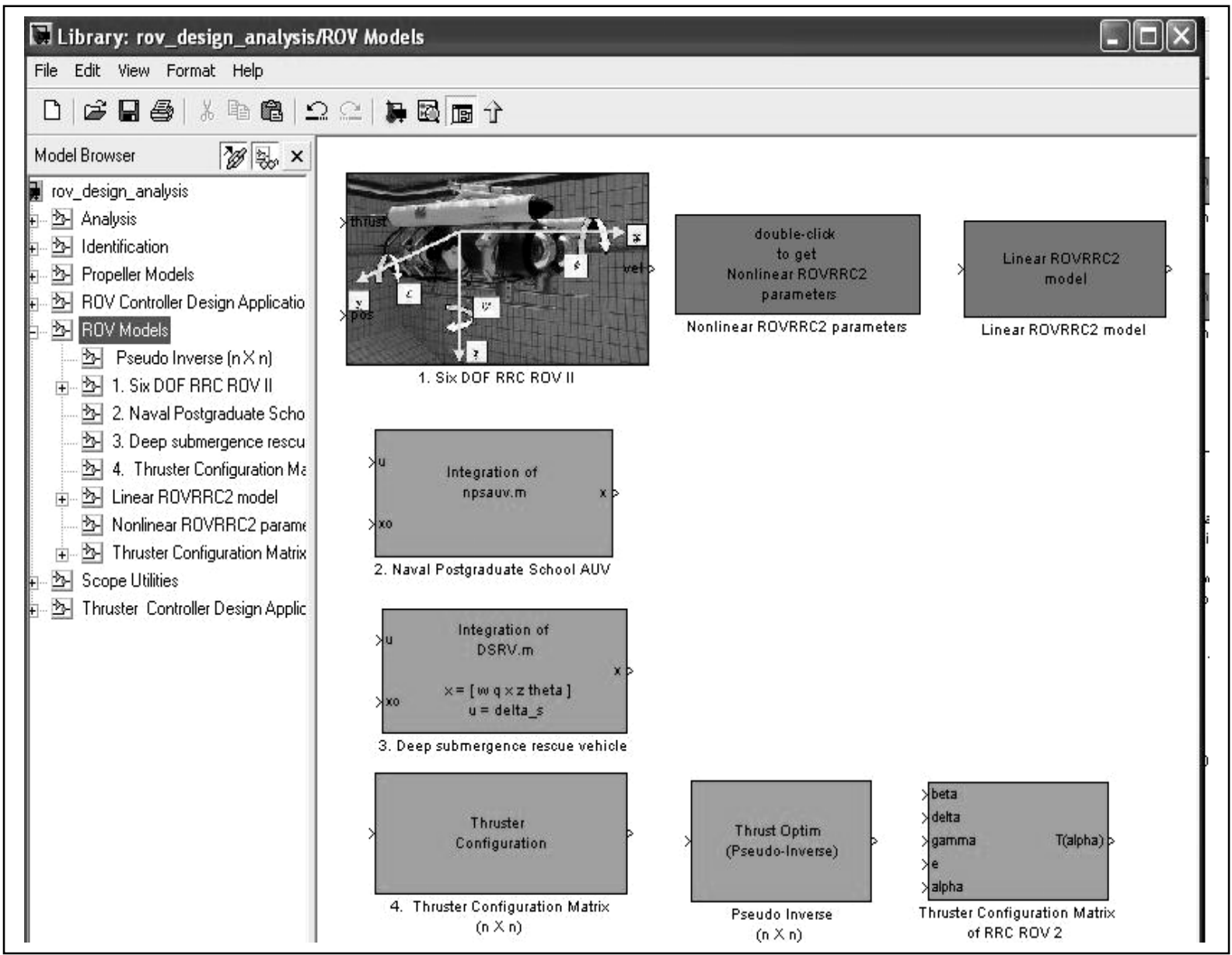

Figure 4: SIMULINK ${ }^{\mathrm{TM}}$ Library Browser Showing ROV Modelling Module.

\subsection{Thruster Controller Design Application and Scope Module}

This Thruster Controller Design library contains the basic SIMULINK ${ }^{\mathrm{TM}}$ blocks that allow various thruster controller designs. The analysis involve the:

- Propeller's shaft speed control.

- Combined propeller's power and torque control

On the other hand, the Scope Utilities Module contains the basic SIMULINK ${ }^{\mathrm{TM}}$ blocks that allow various modelling function to be included in control system design. These blocks include the followings:

- Euler transformation

- Pipeline tracking profile in automatic and manual mode

- Six DOF comparison for responses

- Unit conversion

- Output scope 


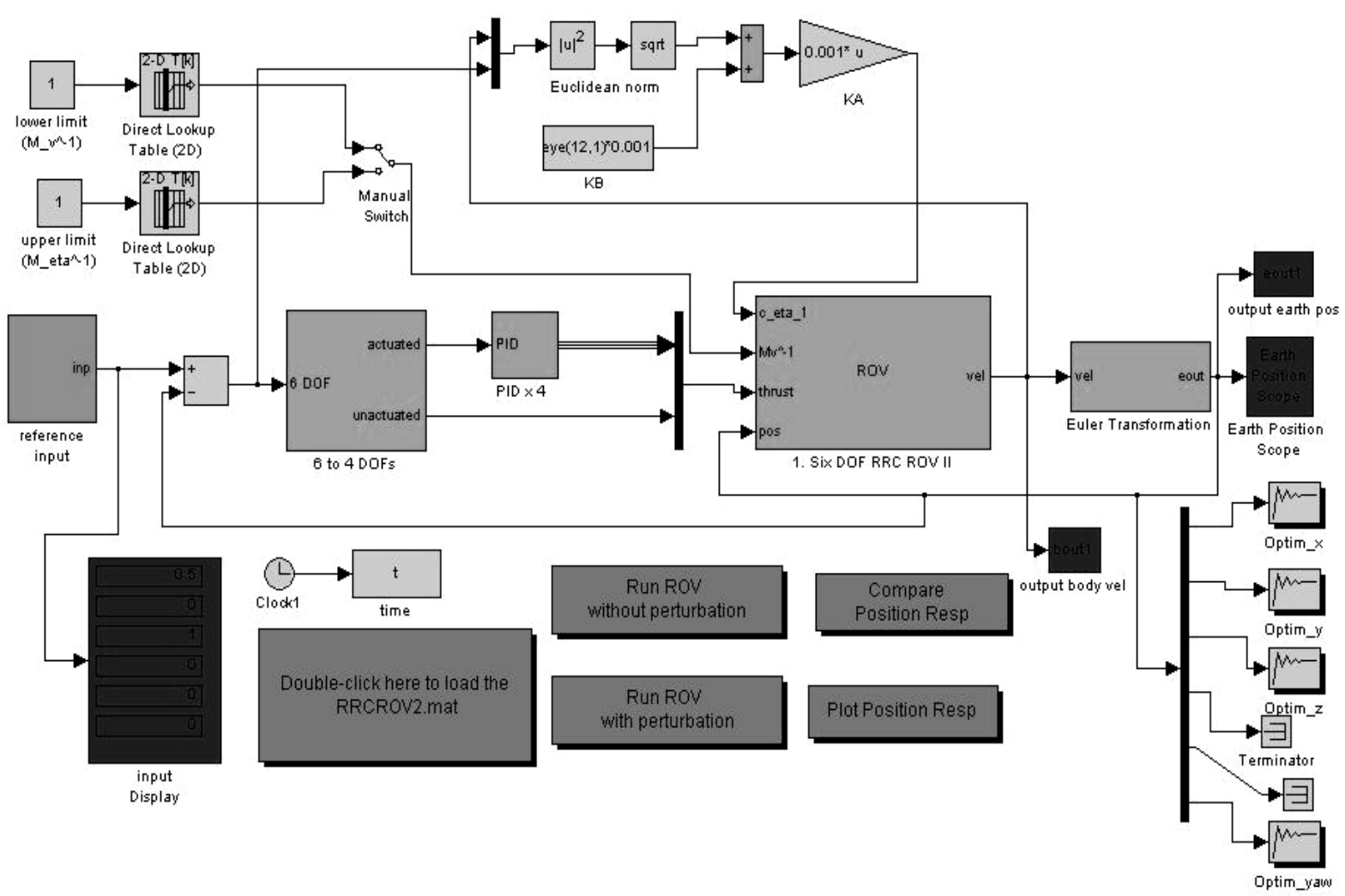

Figure 5: SIMULINK ${ }^{\mathrm{TM}}$ Blocks for PID Controller.
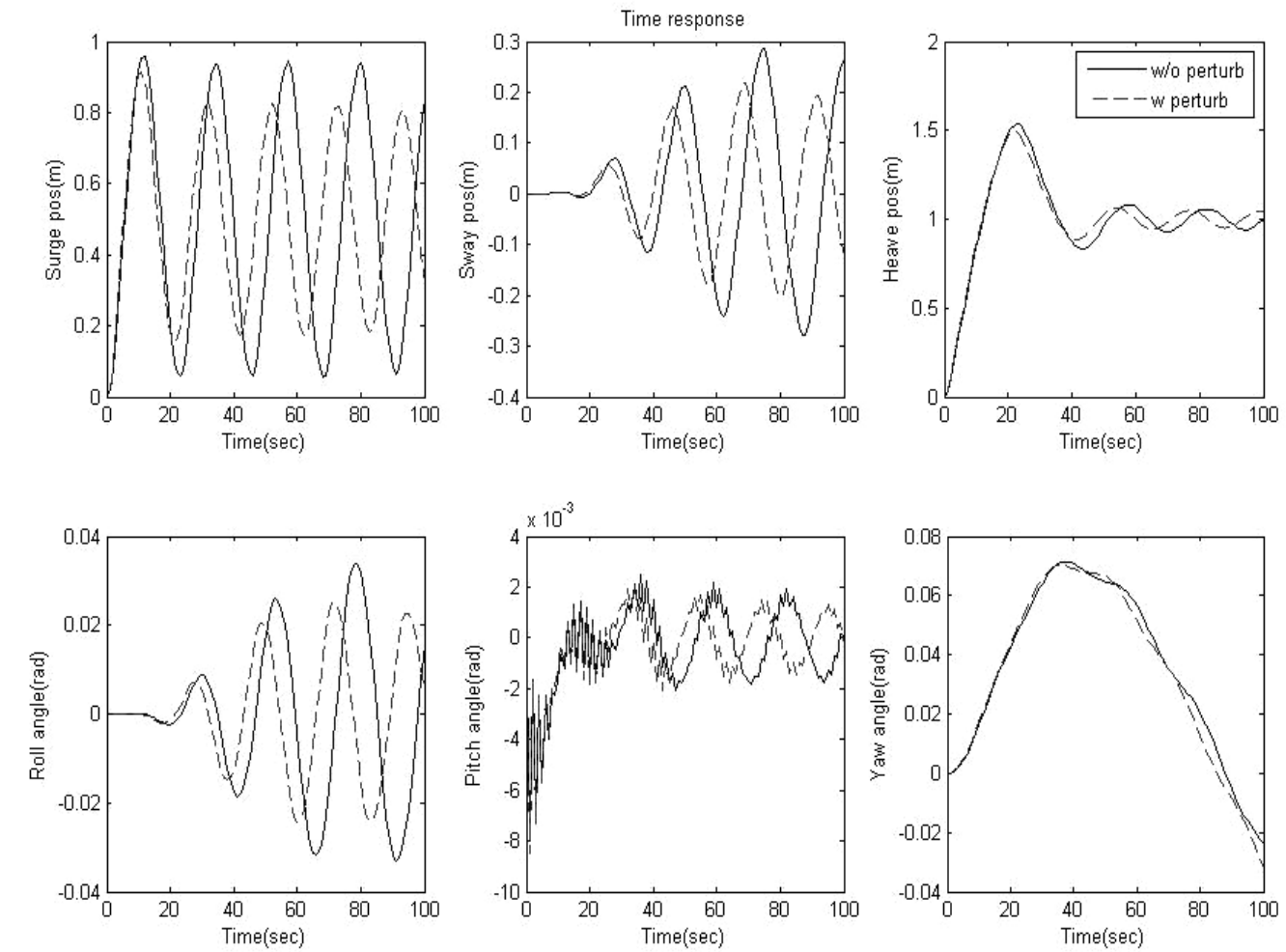

Figure 6: Position Output of ROV Using PID (Reference Input: $x=0.5 \mathrm{~m}$ and $\mathrm{z}=1 \mathrm{~m}$ ). 


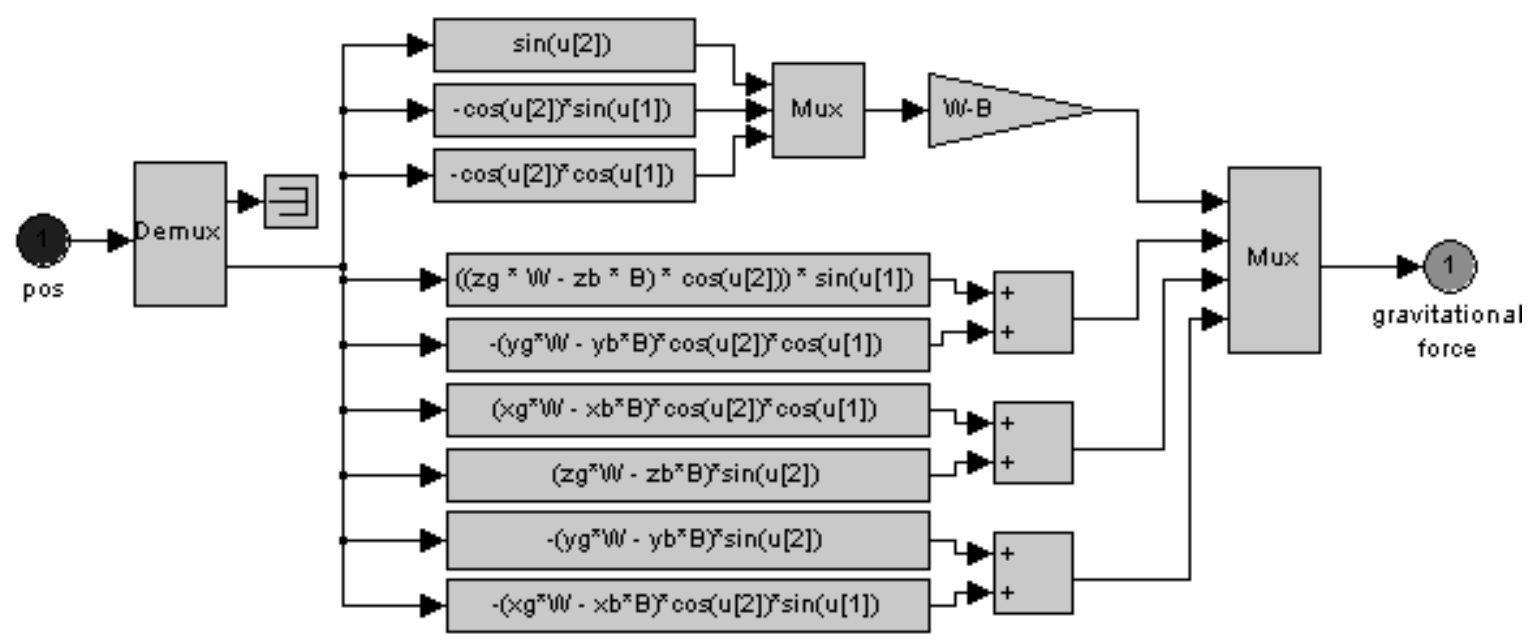

Figure 7: SIMULINK ${ }^{\mathrm{TM}}$ Blocks Showing the $g\left(\eta_{2}\right)$ Mathematical Model.

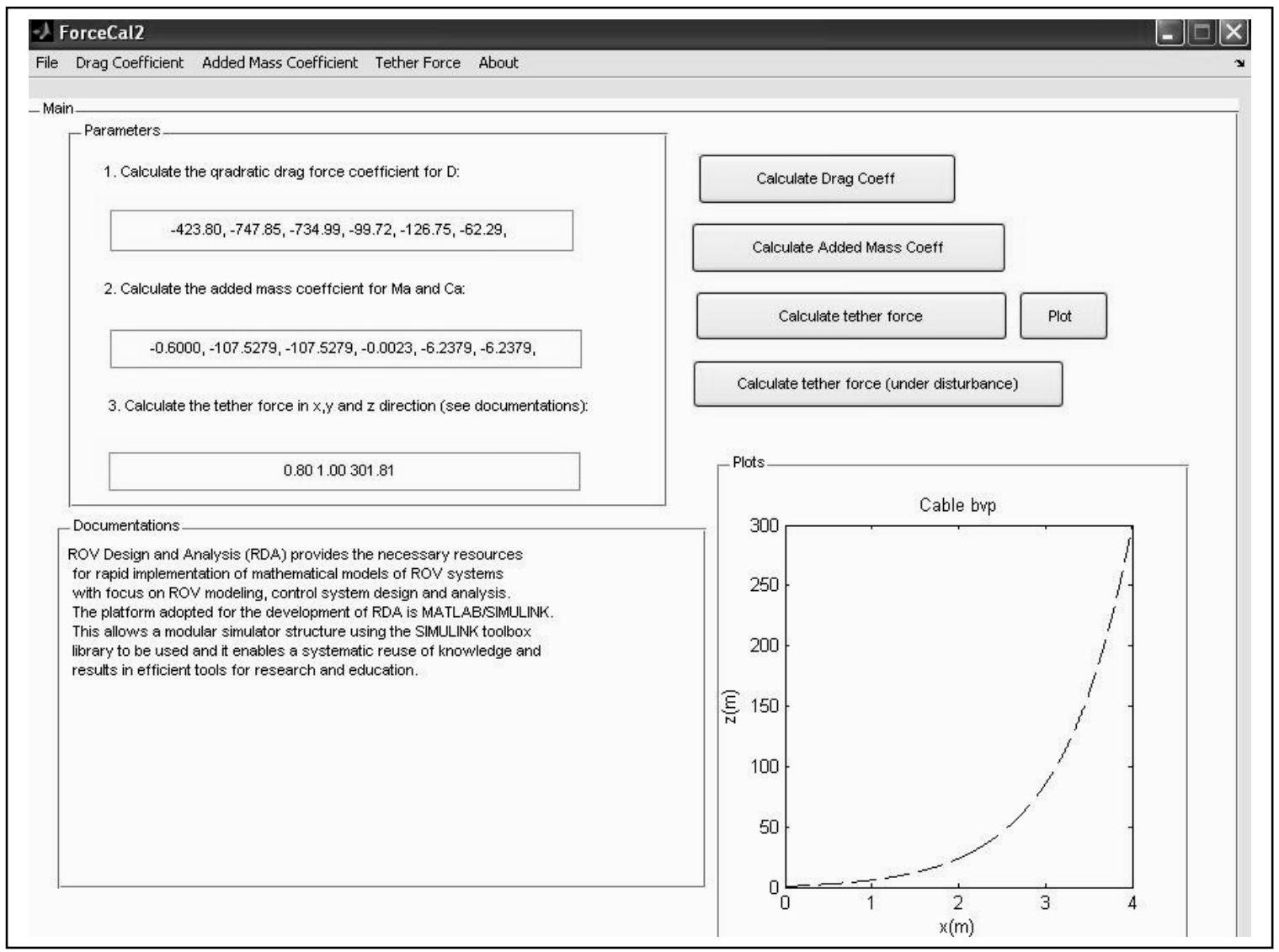

Figure 8: GUI to Determine ROV RRC II Parameters.

\section{CONCLUSIONS}

This paper considers the dynamic modelling and simulation, in particular the RRC ROV II using RDA toolbox. As mentioned, extension to other type of ROV model is available in the ROV Model module. We have provided a brief derivation of the ROV model required for simulation and followed by an overview of RDA package designed under MATLAB/ SIMULINK $^{\mathrm{TM}}$ environment that included the software structure and its respective module 
features. An example on PID control was shown to demonstrate the use of the Controller Design module that contains other module components such as Euler transformation blocks and other utilities. This closed-loop control system design using PID give a fairly good output performance even with the presence of the parameter perturbation (due to the mass inertia and Centripetal and coriolis matrix). A 2D-lookup table was used to model these disturbances. Instead of using the trial and error method, the PID parameters can be conveniently tuned by the Response Optimization toolbox in the MATLAB/SIMULINK. In summary, the RDA provides a systematic methodology in control system simulation and analysis before implementation under best-to-knowledge environment.

For future work, more controller scheme such as hybrid and nonlinear controller design will be incorporated in the package. For more accurate ROV modelling, the simulation package will integrate with the computational fluid dynamic results from WAMIT [16] that provides the hydrodynamic force coefficient.

\section{REFERENCES}

[1] Fossen, T. I. (2000). Marine Control Systems: Guidance, Navigation and Control of Ships, Rigs and Underwater Vehicles, Marine Cybernetics AS. (Free Software: Matlab GNC Toolbox)

[2] Fossen, T. I. (1994). Guidance and control of ocean vehicles, New York-Wiley

[3] Koh, T. H.; Lau, M. W. S.; Low, E.; Seet, G. G. L.; Cheng, P. L. (2002). Preliminary studies of the modelling and control of a twin-barrel underactuated underwater robotic vehicle, ICARCV, 1043-1047

[4] Lau, M. W. S.; Swei, S. S. M.; Seet, G. G. L.; Low, E.; Cheng, P. L. (2003). Control of an underactuated remotely operated underwater vehicle, Proceeding of IMech Engineering, 343-358

[5] Fossen, T. I.; Fjellstad, O. E. (1995). Nonlinear modelling of marine vehicles in 6 degrees of freedom, Journal of Mathematical Modelling of Systems, Vol. 1, No. 1

[6] Potter, M. C.; Wiggert, D. C. (1991). Mechanics of fluids, Prentice-Hall, Englewood Cliffs, New Jersey

[7] Cheng, P. L.; Lau, M. W. S.; Low, E.; Seet, G. G. L. (1999). Modelling of a thruster for an underwater robotic vehicle, Proceedings of the $11^{\text {th }}$ International Symposium on Unmanned Untethered Submersible Technology (UUST'99), Autonomous Undersea Systems Institute, New Hampshire, 455-466

[8] Fossen, T. I.; Blanke, M. (2000). Nonlinear output feedback control of underwater-vehicle propellers using feedback form estimated axial flow velocity, IEEE Journal of Oceanic Engineering, Vol. 25, No.2

[9] Smallwood, D. A.; Whitcomb, L. L. (2004). Model based dynamic positioning of underwater robotic vehicles: theory and experiment, IEEE Journal of Oceanic Engineering, Vol. 29, No. 1

[10] Fossen, I.; Sagatun, S. I. (1991). Adaptive control of nonlinear systems: A case study of underwater robotic systems, Journal of Robotic Systems, JRS-8

[11] Kaminer, I.; Pascoal, A. M.; Silvestre, C. J.; Khargonekar, P. P. (1991). Control of an underwater vehicle using the h-infinity synthesis, Proceedings of the 30th Conference on Decision and Control, Brighton, England, 2350-2355

[12] Rodrigues, L.; Tavares, P.; Prado, M. (1996). Sliding mode control of an auv in the diving and steering plane, Proceedings of Ocean'96, Coastal Ocean Prospect for the 21st Century by the Marine Technical Society

[13] Chellabi, A.; Nahon, M. (1995). Feedback Linearisation of Undersea Vehicles, Proceedings of OCEANS, 1410-1415

[14] Antonelli, G.; Chiaverini, S.; Sarkar, M.; West, M. (2001). Adaptive control of an autonomous underwater vehicle: experimental results on ODIN, IEEE Transactions on Control Systems Technology, Vol. 9, No. 5, 756-765

[15] Chin, C. S., Munro, N. (2003) Control of the ALSTOM Gasifier Benchmark Problem using $\mathrm{H}_{2}$ Methodology, Journal of Process Control, Vol. 13, No. 8, 759-768

[16] WAMIT Inc. WAMIT User manual, from http://www.wamit.com, accessed on 23-07-2006 